\title{
Effect of lattice strain on hydrogen diffusion in Pd: A density functional theory study
}

\author{
Henrik Grönbeck ${ }^{1, *}$ and Vladimir P. Zhdanov ${ }^{1,2}$ \\ ${ }^{1}$ Department of Applied Physics and Competence Centre for Catalysis, Chalmers University of Technology, SE-412 96 Göteborg, Sweden \\ ${ }^{2}$ Boreskov Institute of Catalysis, Russian Academy of Sciences, Novosibirsk 630090, Russia \\ (Received 29 March 2011; revised manuscript received 29 May 2011; published 3 August 2011)
}

\begin{abstract}
The density functional theory is used to study the effect of lattice strain on hydrogen diffusion in Pd. The activation energy for this process is found to increase dramatically with increasing compressive lattice strain. In particular, the activation energy is close to double for an isotropic compression of 5\% both in the $\alpha$ and $\beta$ phases. For tensile strain, the activation energy is instead decreased. This finding has important consequences for the interpretation of various kinetic processes occurring with participation of hydrogen and other interstitial atoms in macroscopic solid samples and nanoparticles.
\end{abstract}

DOI: 10.1103/PhysRevB.84.052301

PACS number(s): 66.30.jp, 62.20.-x, 66.30.Pa, 88.30.R-

Diffusion of interstitial atoms in solids is a basic kinetic process that governs various phenomena such as phase transitions, formation of cracks, and chemical reactions. It can be observed under environmental conditions and is relevant to numerous applications where microelectronics, new energy materials (hydrogen storage), reactor safety (hydrogen-induced embrittlement), and sensors are some examples. From a fundamental point of view, the process is of high interest owing to the complex interplay between the diffusion and self-induced and/or external lattice strain.

If the concentration of interstitial atoms, $c$, is low and the lattice strain is negligible, the diffusion is described by Fick's laws. In particular, the diffusion flux, $J$, of atoms is given by

$$
J=-D \nabla c,
$$

where $D$ is the diffusion coefficient. Under more complex conditions that include strain and/or high concentrations, the diffusion flux can phenomenologically be expressed by

$$
J=-B c \nabla \mu,
$$

where $\mu$ is the chemical potential of atoms, and $B$ is the transport coefficient. In the absence of lattice strain, Eq. (2) should be equivalent to Eq. (1) provided that $c$ is low. Taking into account that in this limit $\mu=k_{B} T \ln (c)+$ const, one accordingly has $B=D / k_{B} T$. The contribution of lattice strain to the chemical potential is phenomenologically represented as

$$
\Delta \mu=-\frac{v_{\circ} \sigma_{i i}}{3},
$$

where $v_{\circ}$ is the increment of the lattice volume per interstitial atom, and $\sigma_{i i}$ is the trace of the stress tensor. The corresponding contribution to the diffusion flux is given by

$$
J_{s}=\frac{D c v_{\circ} \nabla \sigma_{i i}}{3 k_{B} T} .
$$

This approach [Eqs. (3) and (4)] has been widely used in material science since the seminal studies by Cahn and Larche $^{1,2}$ (see, e.g., recent articles ${ }^{3}$ and references therein).

Appreciable lattice strain may influence $D$. Although this effect was noticed two decades ago, ${ }^{2}$ its quantitative role has not been explored. The linear corrections proposed in Ref. 2 are applicable only in situations when their role is minor and this factor is generally ignored in applications. ${ }^{3}$ Phenomenologically, the dependence of $D$ on lattice strain can be described in the framework of the transition-state theory (TST) implying that the diffusion jumps are activated. In particular, expression (3) represents the shift of the energy of the initial state for diffusion, and a similar expression can be used for the shift of the energy at the transition state. ${ }^{4}$ Following this line yields

$$
D=D_{\circ} \exp \left[-\frac{\left(v_{\circ}-v_{*}\right) \sigma_{i i}}{3 k_{B} T}\right],
$$

where $D_{\circ}$ is the diffusion coefficient at $\sigma_{i i} \rightarrow 0$, and $v_{*}$ is the volume increment that corresponds to the transition state. Equation (5) indicates that lattice strain may either suppress or facilitate diffusion depending on the sign of the difference $v_{\circ}-v_{*}$. It, however, does not allow determination of the sign and value of the effect.

In this Brief Report, we use density functional theory (DFT) calculations to investigate the effect of lattice strain on activated interstitial diffusion. As a generic example, $\mathrm{H}$ diffusion in $\mathrm{Pd}$ is analyzed. This system is of particular interest, because $\mathrm{H}$ absorption in $\mathrm{Pd}$ is often accompanied by hydride formation. Specifically, at $T<T_{c}$, $\mathrm{H}$ is well known to be observed in the diluted state ( $\alpha$ phase) and/or as hydride ( $\beta$ phase) where the $\mathrm{H}$ loading (with respect to $\mathrm{Pd}$ ) is about or higher than $0.6 .^{5} T_{c} \simeq 565 \mathrm{~K}$ for bulk samples whereas $T_{c}$ is appreciably reduced for nanoparticles. In fact, the hydride formation in $\mathrm{Pd}$ is one of the best examples of first-order phase transitions in solids ${ }^{5,6}$ (for details of such phase transitions, see Ref. 7).

Taking into account that the hydride formation and decomposition occur at relatively high temperatures and bearing in mind the numerous related applications, we focus on $\mathrm{H}$ diffusion treated as a classical activated process in the framework of TST. Experimental studies indicate that in $\mathrm{Pd}$, this regime of diffusion is observed at temperatures above $230 \mathrm{~K}^{8}{ }^{8}$ (For the theory of tunnel diffusion, see, e.g., Ref. 9 and references therein.)

DFT has previously been used extensively to describe various aspects of $\mathrm{H}$ adsorption on the surface and absorption in the bulk of Pd (see Refs. 10 and 11, respectively). The effect of lattice strain on $\mathrm{H}$ diffusion has, however, not been analyzed. The related DFT studies ${ }^{12}$ are focused on the influence of lattice strain on the binding and dissociation energies of adsorbed surface species. The corresponding results are, however, not directly applicable to diffusion of interstitial 
atoms in solids in general and $\mathrm{H}$ diffusion in the bulk of $\mathrm{Pd}$ in particular.

Herein, the DFT is applied in the implementation with plane waves and pseudopotentials. ${ }^{13,14}$ The Perdew-BurkeErnzerhof (PBE) parametrization is used for the approximation of the exchange-correlation (xc) functional. ${ }^{15}$ Ultrasoft scalar-relativistic pseudopotentials (generated with PBE) are employed to describe the interaction between the valence electrons and the core. ${ }^{16} \mathrm{Pd}$ is treated with a valance of 10. A plane-wave kinetic energy cutoff of $340 \mathrm{eV}$ is used to expand the Kohn-Sham orbitals. Tests with higher cutoff energies show that the relative energies and lattice constants are converged within $5 \mathrm{meV}$ and $0.001 \AA$, respectively. Reciprocal space integration over the Brillouin zone is approximated with finite sampling of the $k$-points. ${ }^{17}$ In particular, 14 special $k$-points were employed in the cubic $(2 \times 2 \times 2)$ cell of the original unit cell.

The calculated lattice constant for the Pd fcc bulk phase is $3.93 \AA$. This is a slight expansion (1\%) with respect to the experimental value $(3.89 \AA)$ and can be attributed to the applied xc-functional. ${ }^{18}$ Gas-phase $\mathrm{H}_{2}$ and atomic $\mathrm{H}$ were treated in a cubic cell with a side of $12 \AA$. The binding energy (bond distance) of $\mathrm{H}_{2}$ was calculated to be $4.53 \mathrm{eV}(0.75 \AA)$, which is in full agreement with previous calculations. ${ }^{15}$ The $\beta$ phase of $\mathrm{PdH}$ has a $\mathrm{NaCl}$ lattice with $\mathrm{H}$ placed in the octahedral position of the $\mathrm{Pd}$ lattice. The lattice constant of $\mathrm{PdH}_{1}$ was calculated to be $4.11 \AA$. The experiment ${ }^{19}$ yields $4.09 \AA$ for this stoichiometry. The heat of formation (with respect to $\mathrm{Pd}$ in the bulk phase and $\mathrm{H}_{2}$ in the gas phase) for $\mathrm{PdH}_{1}$ is calculated to be $-0.23 \mathrm{eV}$. This is in good agreement with the experimental value of $-0.20 \mathrm{eV}^{20}$

The $\mathrm{H}$ absorption is investigated in a cubic $(2 \times 2 \times 2)$ cell of the original unit cell. This is a system with $32 \mathrm{Pd}$ atoms. To ensure that the choice of computational cell is large enough to capture the structural relaxations [this effect is important for the calculation of diffusion barriers ${ }^{11}$ ], $\mathrm{H}$ absorption in the octahedral and tetrahedral sites was evaluated in $\mathrm{a}(3 \times 3 \times 3)$ cell. This cell includes $108 \mathrm{Pd}$ atoms. In this case, four special $k$-points were used. The energies of solution were affected less than $0.01 \mathrm{eV}$ by the increase of the computational cell, which confirms that the $(2 \times 2 \times 2)$ cell in this case is sufficient.

Hydrogen is preferably absorbed in the octahedral $(\mathrm{O})$ sites of the Pd fcc lattice. Upon diffusion between O-sites, the $\mathrm{H}$ atom passes through a tetrahedral $(\mathrm{T})$ site (the local geometries of these sites are shown in Fig. 1). To quantify this pathway in the dilute $\alpha$ phase, we have calculated (Fig. 1) the $\mathrm{H}$ absorption potential energy (with respect to $\mathrm{H}_{2}$ in the gas phase), the difference in total energies at the $\mathrm{O}$ and $\mathrm{T}$ sites, and the barrier for $\mathrm{H}$ diffusion as a function of isotropic lattice compression. The $\mathrm{H}$ absorption potential energy at zero compression (with the optimal Pd lattice constant) is found to be $-0.21 \mathrm{eV}$. The absorption potential energy is close to linearly increasing as the lattice is uniformly strained [the slope of this curve, $11.6 \mathrm{eV}$, is in fair agreement with that, $8.1 \mathrm{eV}$, predicted by Eq. (3) with the experimentally measured parameters]. The difference of the total energies at the $\mathrm{O}$ - and T-sites $(0.07 \mathrm{eV}$ at zero compression) shows a linear dependence as well. The positive absorption potential energies at high enough compressive strain indicates that this is a metastable situation which is mainly relevant under transient conditions.

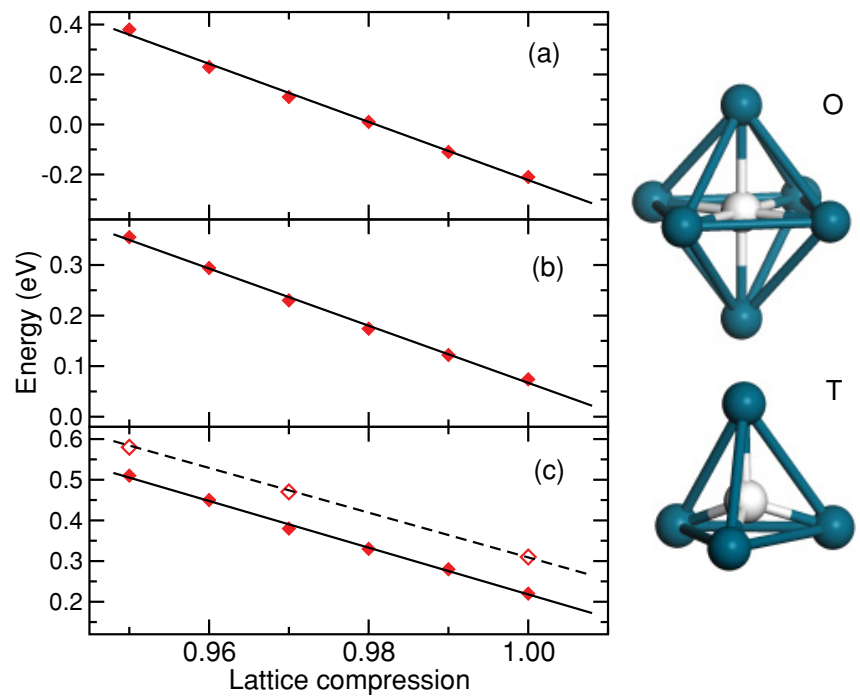

FIG. 1. (Color online) Energetics for $\mathrm{H}$ diffusion in $\alpha-\mathrm{Pd}$ as a function of lattice compression: (a) $\mathrm{H}$ absorption potential energy at the octahedral site, (b) difference of the total energies at the $\mathrm{O}$ - and the $\mathrm{T}$-sites, and (c) activation energy for $\mathrm{O}$ to $\mathrm{T}$ diffusion. Open symbols in (c) are activation energies with the zero-point energy corrections. The local geometries of the O- and T-sites are shown to the right. Atomic color codes: Pd (dark) and $\mathrm{H}$ (white).

The strain-related reduction of the $\mathrm{H}$ absorption potential energy, which previously has been predicted for $\mathrm{H}$ in bcc-Fe, ${ }^{21}$ can be understood from the increased Pauli and electrostatic repulsion as the $\mathrm{H}-\mathrm{Pd}$ distances are reduced. The repulsive interaction can be described by using an exponential pairwise potential. ${ }^{22}$ Close to the equilibrium, the Taylor expansion of the repulsive potential has leading order linear and quadratic terms in the distance dependence. The increased difference of the $\mathrm{H}$ absorption energies at the $\mathrm{O}$ - and T-sites, as the lattice is compressed, comes from the fact that $\mathrm{H}$ in the T-site is closer to the metal atoms. The $\mathrm{H}-\mathrm{Pd}$ distance in the $\mathrm{O}$-site at zero compression is $1.98 \AA$, whereas it is $1.77 \AA$ in the T-site.

For the optimized Pd lattice, the activation energy for $\mathrm{H}$ diffusion [Fig. 1(c)] is found to be $0.22 \mathrm{eV}$. This is close to the experimental value [0.20-0.23 eV (Ref. 8)] as well as previous theoretical reports. ${ }^{11}$ There is a marked dependence of the barrier on the lattice stain. In particular, the barrier is close to double $(0.52 \mathrm{eV})$ for a compression of $5 \%$. The barrier is in all cases close to the point where $\mathrm{H}$ is passing through the triangle that separates the $\mathrm{O}$ - and T-sites. For the relaxed lattice, the $\mathrm{H}-\mathrm{Pd}$ distance at the transition state is calculated to be $1.70 \AA$. The strong strain dependence on the diffusion barrier can be rationalized in the same way as the energy differences between the O- and T-sites.

The activation barriers reported above do not include the zero-point energy (ZPE) effects. To estimate the corresponding contribution to the activation energy, the vibrational modes of $\mathrm{H}$ are evaluated with a frozen metal lattice at the $\mathrm{O}$-site (three modes) and the transition state (two modes), respectively. Inclusion of the ZPE effects yields [Fig. 1(c)] an increased diffusion barrier by $\sim 0.08 \mathrm{eV}$ that is the same over the range of investigated lattice compressions. Also the role of tunneling in the diffusion is estimated. At temperatures above room temperature, the tunneling increases the diffusion 
coefficient (as compared to the TST value) by a factor of $\chi=\hbar \omega /\left[2 k_{B} T \sin \left(\hbar \omega / 2 k_{B} T\right)\right]$, where $\omega$ is the imaginary frequency at the transition state. ${ }^{23}$ With the calculated frequencies for the unconstrained $\left(361 \mathrm{~cm}^{-1}\right)$ and $5 \%$ compressed $\left(462 \mathrm{~cm}^{-1}\right)$ lattice at $T=300 \mathrm{~K}, \chi$ is calculated to be 1.14 and 1.17, respectively. As expected, the role of tunneling is minor at this or higher temperatures and shows a weak dependence on lattice compression.

The experimental stoichiometry of the $\beta$ phase is known to be $\mathrm{PdH}_{x}$ with $x \approx 0.7$, which therefore represents the relevant composition for studies of $\mathrm{H}$ diffusion. As the system has an uncertainty with respect to the choice of $\mathrm{O}$ sites for occupation by $\mathrm{H}$, we explore 75 different configurations generated by random occupation of $22 \mathrm{O}$ sites in the $(2 \times 2 \times 2)$ cell (i.e., for $\mathrm{Pd}_{32} \mathrm{H}_{22}$ ). These calculations are performed in a Pd cell that structurally corresponds to the equilibrium lattice of $\mathrm{PdH}_{1}$. The differences in total energies for the investigated configurations exhibit a narrow energy distribution where the highest and the lowest energies are separated by only $0.19 \mathrm{eV}(0.01 \mathrm{eV}$ per $\mathrm{H}$ atom). A configuration at the mean energy is chosen for further investigations of $\mathrm{H}$ diffusion. The lattice constant is optimized for this structure and found to be $4.06 \AA$, which is close to the experiment $\left(4.04 \AA^{19}\right)$.

To analyze $\mathrm{H}$ diffusion in the $\beta$ phase, the same calculations are performed (Fig. 2) as for the $\alpha$ phase. Just as for the $\alpha$ phase, the difference in total energy of the O- and T-sites and the diffusion barrier is found to be linearly dependent of the lattice strain. In fact, the barriers are nearly identical for the two phases. The dependence of the $\mathrm{H}$ absorption energy for the $\beta$ phase is, however, nonlinear. This comes from the fact that zero compression represents the equilibrium lattice for $\mathrm{Pd}_{32} \mathrm{H}_{22}$.

The key finding of the present study is that the activation energy for $\mathrm{H}$ diffusion in $\mathrm{Pd}$ dramatically increases with

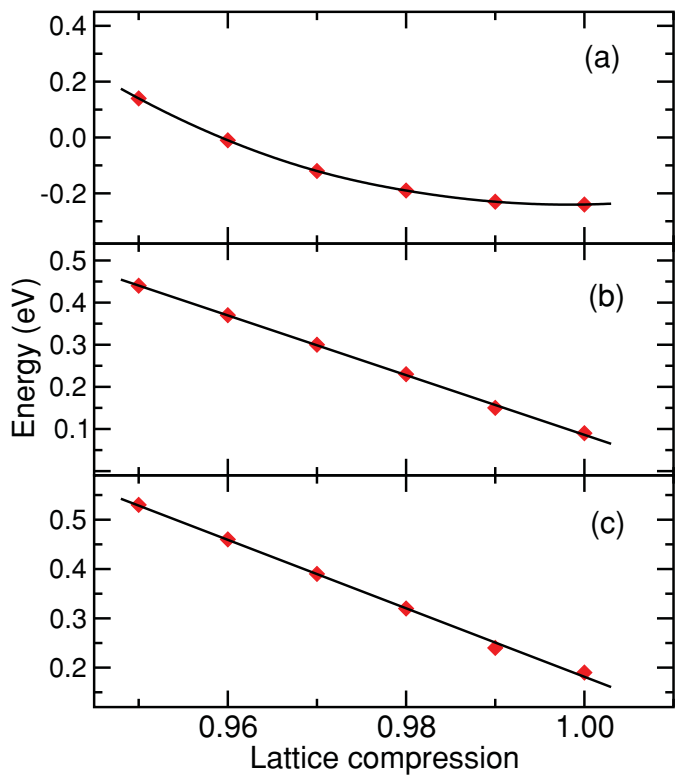

FIG. 2. (Color online) Energetics for $\mathrm{H}$ diffusion in $\mathrm{Pd}_{32} \mathrm{H}_{22}$ as a function of lattice compression: (a) average $\mathrm{H}$ absorption potential energy, (b) difference of the $\mathrm{H}$ energies at the $\mathrm{O}$ - and the $\mathrm{T}$-sites sites, and (c) activation energy for $\mathrm{O}$ to $\mathrm{T}$ diffusion.

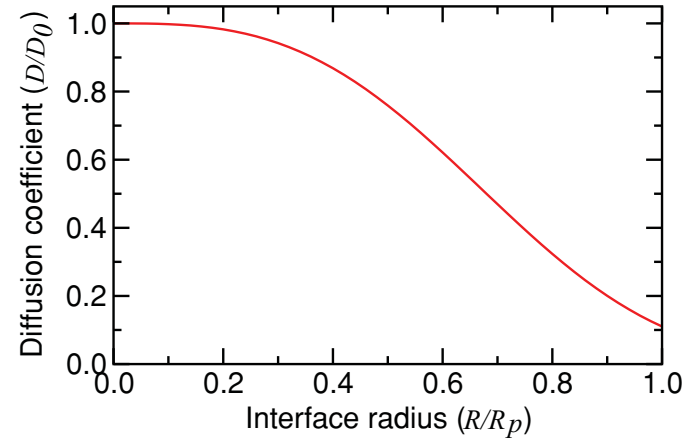

FIG. 3. (Color online) Coefficient of $\mathrm{H}$ diffusion in the hydride shell of a spherical $\mathrm{Pd}$ nanoparticle as a function of the interface radius at $300 \mathrm{~K}$. The results are for $\mathrm{PdH}_{0.7}$ with $C=2.3 \mathrm{eV}$ and $\kappa=0.032$ (these parameters were obtained from the present DFT calculations; see, e.g., Fig. 2).

increasing compressive lattice strain. For the tensile strain, the effect is opposite. In particular, the activation energy is close to double for an isotropic compression of $5 \%$ in both phases. This finding has important consequences for the interpretation of various kinetic processes that occur in macroscopic Pd samples and nanoparticles.

One important example is hydride formation in $\mathrm{Pd}$ nanoparticles. This process is expected to be limited by $\mathrm{H}$ diffusion via a hydride shell formed on the periphery of the nanoparticle. ${ }^{24}$ The activation energy for $\mathrm{H}$ diffusion in the hydride can be written as $E_{a}=E_{a}^{\circ}-A \sigma_{i i}$, where $E_{a}^{\circ}$ is the value that corresponds to the stress-free case, and $A$ is a constant [cf. Eq. (5)]. Taking into account that the trace of the stress tensor is proportional to the trace of the deformation tensor, $u_{i i}$, this expression can be rewritten as $E_{a}=E_{a}^{\circ}-C u_{i i}$, where $C$ is a constant (this constant can be calculated by the use of the results reported in Fig. 3). The deformation tensor for the self-induced strain in the hydride shell of a spherical nanoparticle is given by $u_{i i}=-2 \kappa(1-2 \sigma) R^{3} /\left[(1-\sigma) R_{p}^{3}\right]{ }^{4}$ where $R_{p}$ is the particle radius, $R\left(0 \leqslant R \leqslant R_{p}\right)$ is the coordinate that corresponds to the interface between the two phases, $\kappa$ is the linear mismatch parameter, and $\sigma$ is Poisson's ratio ( 0.38 for $\mathrm{Pd})$. In addition, there is strain related to surface tension. The latter strain is, however, nearly constant during the formation of the hydride phase and accordingly can be omitted here. Thus, the diffusion coefficient can be described by

$$
D=D_{\circ} \exp \left[-\frac{2 C \kappa(1-2 \sigma) R^{3}}{(1-\sigma) R_{p}^{3} k_{B} T}\right],
$$

where $D_{\circ}$ is the value that corresponds to $R \rightarrow R_{p}$. By the use of expression (6), $D$ has been calculated for $\mathrm{H}$ diffusion in the hydride shell as a function of $R$ (Fig. 3). The diffusion coefficient is lowered substantially in the beginning of the hydration (at $R \rightarrow R_{p}$ ). In fact, at this radius, the diffusion coefficient is reduced to only $10 \%$ of the strain-free case. This feature, which is related to compression of the hydride shell due to its contact with the metal core, qualitatively changes the course of the hydration kinetics by extending the initial phase. 
To conclude, we have used DFT calculations to investigate the effect of lattice strain on activated interstitial hydrogen diffusion in Pd. The effect is found to be dramatic in the $\alpha$ phase as well as in the $\beta$ phase. By coupling the first principles results to a continuum model for hydrogen diffusion in $\mathrm{Pd}$ nanoparticles, it is demonstrated that the self-induced strain may qualitatively change the hydration kinetics. As the $\mathrm{H} / \mathrm{Pd}$ system is generic, similar effects are anticipated in many other situations and/or systems.

Support from the Swedish Research Council (HG) and the Swedish Energy Agency (VPZ, NANO-SEE 181-1) is acknowledged. The calculations were performed at $\mathrm{C} 3 \mathrm{SE}$ (Göteborg). *ghj@chalmers.se

${ }^{1}$ J. W. Cahn and F. C. Larche, Acta Metall. 30, 1835 (1982).

${ }^{2}$ F. C. Larche and P. W. Voorhees, Def. Diff. Forum 129, 31 (1996).

${ }^{3}$ F.-Z. Xuan, S.-S. Shao, Z. Wang, and S.-T. Tu, J. Phys. D: Appl. Phys. 42, 015401 (2009); Thin Solid Films 518, 4345 (2010); P. Zoltowski, Electrochim. Acta 55, 6274 (2010); H. Haftbaradaran, J. Song, W. A. Curtin, and H. Gao, J. Power Sources 196, 361 (2011).

${ }^{4}$ V. P. Zhdanov, Chem. Phys. Lett. 492, 77 (2010).

${ }^{5}$ M. Yamauchi, H. Kobayashi, and H. Kitagawa, ChemPhysChem 10, 2566 (2009).

${ }^{6}$ A. Pundt and R. Kirchheim, Annu. Rev. Mater. Sci. 36, 555 (2006).

${ }^{7}$ R. B. Schwarz and A. G. Khachaturyan, Phys. Rev. Lett. 74, 2523 (1995).

${ }^{8}$ Hydrogen in Metals III, edited by H. Wipf (Springer, Berlin, 1986), p. 63.

${ }^{9}$ P. G. Sundell and G. Wahnström, Phys. Rev. Lett. 92, 155901 (2004).

${ }^{10}$ M. P. Jigato, B. Coussens, and D. A. King, J. Chem. Phys. 118, 5623 (2003); V. Pallassana, M. Neurock, L. B. Hansen, B. Hammer, and J. K. Nørskov, Phys. Rev. B 60, 6146 (1999); N. Lopez, Z. Lodziana, F. Illas, and M. Salmeron, Phys. Rev. Lett. 93, 146103 (2004).

${ }^{11}$ C. T. Chan and S. G. Louie, Phys. Rev. B 27, 3325 (1983); C. Elsässer, K. M. Ho, C. T. Chan, and M. Fähnle, ibid. 44, 10377 (1991); W. Zhong, Y. Cai, and D. Tomanek, ibid. 46, 8099 (1992); K. G. McLennan, E. M. Gray, and J. F. Dobson, ibid. 78, 014104 (2008); H. M. Lawler and D. R. Trinkle, ibid. 82, 172101 (2010).

${ }^{12}$ M. Mavrikakis, B. Hammer, and J. K. Norskov, Phys. Rev. Lett. 81, 2819 (1998); Y. Uesugi-Saitow and M. Yata, ibid. 88, 256104 (2002); M. Yata, Y. Uesugi-Saitow, M. Kitajima, A. Kubo, and V. E. Korsukov, ibid. 91, 206103 (2003); M. Yata, Phys. Rev. B 81, 205402 (2010).

${ }^{13}$ S. J. Clark, M. D. Segall, C. J. Pickard, P. J. Hasnip, M. J. Probert, K. Refson, and M. C. Payne, Zeitchr. Kristal. 220, 567 (2005).

${ }^{14}$ We use the CASTEP program.

${ }^{15}$ J. P. Perdew, K. Burke, and M. Ernzerhof, Phys. Rev. Lett. 77, 3865 (1996).

${ }^{16}$ D. Vanderbilt, Phys. Rev. B 41, 7892 (1990).

${ }^{17}$ H. J. Monkhorst and J. D. Pack, Phys. Rev. B 13, 5188 (1976); J. D. Pack and H. J. Monkhorst, ibid. 16, 1748 (1977).

${ }^{18}$ M. Körling and J. Häglund, Phys. Rev. B 45, 13293 (1992).

${ }^{19}$ J. E. Schriber and B. Morosin, Phys. Rev. B 12, 117 (1975).

${ }^{20}$ Hydrogen in Metals II, edited by G. Alefeld and J. Völkl (Springer, Berlin, 1978), p. 73.

${ }^{21}$ R. Matsumoto, Y. Inoue, S. Taketomi, and M. Miyazaki, Scr. Mater. 60, 555 (2009).

${ }^{22}$ R. P. Gupta, Phys. Rev. B 23, 6265 (1981).

${ }^{23}$ R. P. Bell, The Tunnel Effect in Chemistry (Chapman and Hall, London, 1980).

${ }^{24}$ C. Langhammer, V. P. Zhdanov, I. Zoric, and B. Kasemo, Phys. Rev. Lett. 104, 135502 (2010). 